\title{
Microbial transformation of a tertiary allylalcohol: regioselective isomerisation of linalool to geraniol without nerol formation
}

\author{
Sabine Foß, Jens Harder * \\ Department of Microbiology, Max-Planck-Institut für Marine Mikrobiologie, Celsiusstr. 1, D-28259 Bremen, Germany
}

Received 29 January 1997; accepted 3 February 1997

\begin{abstract}
Linalool is a natural monoterpenoic allylalcohol with a tertiary alcohol group. The initial reaction of linalool degradation under anoxic conditions was studied in nitrate-limited cultures of a recently isolated denitrifying bacterium, strain 47Lol. The primary allylalcohol geraniol was detected in the stationary phase of cultures grown on linalool. Geranial and linalool appeared in the medium of geraniol-fed cultures. Nerol was not isomerised to geraniol or linalool. It was oxidised to neral, but not further degraded. These observations indicate the presence of a new enzyme reaction, a 3,1-hydroxyl- $\Delta^{1}-\Delta^{2}-$ mutase that regioselectively isomerises linalool and geraniol.
\end{abstract}

Keywords: Monoterpene; Biotransformation; Denitrifying bacteria; Tertiary alcohol; Allylic rearrangement

\section{Introduction}

Monoterpenes are natural substances that present a small carbon pool with a high turnover rate in the annual global carbon cycle. Terpen emission from trees is estimated at $4.8 \times 10^{14} \mathrm{~g}$ terpenes/year [1]. Biodegradation by aerobic bacteria involves several mono- and dioxygenases [2,3]. Monoterpenes also enter soil and freshwaters and reach anoxic habitats [4]. There, molecular oxygen is not present and the initial transformation reactions of monoterpenes are unknown.

Linalool (3,7-dimethyl-1,6-octadien-3-ol) is an important fragrance and is produced in large amounts

* Corresponding author. Tel.: +49 (421) 2028 750; fax: +49 (421) 2028 580;

e-mail: jens@postgate.mpi-mm.uni-bremen.de as an intermediate of the synthesis of vitamin A [5]. It contains a non-oxidisable tertiary alcohol and an alkene bond as functional groups. In accordance with the recalcitrant structure of linalool, initial reactions of aerobic biodegradation pathways involve a monooxygenase synthesising 10-hydroxylinalool in bacteria [6] or an epoxidase and a hydrolase yielding 2,6-dimethyl-oct-7-en-2,3,6-triol in fungi [7]. Recently, we observed the formation of geraniol and geranial, but not of nerol in anoxic denitrifying enrichment cultures on linalool as sole electron donor and carbon source [8]. A catalyst for the regioselective isomerisation of linalool to geraniol has not been reported. For industrial geraniol production, linalool is isomerised to a geraniol-nerol mixture using orthovanadate complexes as catalysts and geraniol is separated from nerol by distillation [5]. Hence we studied the fate of linalool, geraniol and nerol in 
cultures of the recently isolated denitrifying strain, 47Lol, to find evidence for a regioselective allylic rearrangement in a pure culture.

\section{Materials and methods}

The denitrifying strain 47Lol has been recently isolated on linalool as sole carbon source and electron donor and nitrate as electron acceptor [8]. Cells were grown in $156-\mathrm{ml}$ serum bottles containing 120 $\mathrm{ml}$ of anoxic medium, $10 \mathrm{ml}$ of 2,2,4,4,6,8,8-heptamethylnonane, a headspace of $\mathrm{N}_{2}-\mathrm{CO}_{2}(90: 10, \mathrm{v}: \mathrm{v})$, $1200 \mu \mathrm{mol}$ nitrate $(10 \mathrm{mM})$ and approximately 240 $\mu$ mol monoterpene ( \pm -linalool, geraniol or nerol). Inoculation occurred with $4 \mathrm{ml}$ of carbon-limited culture recently grown on linalool.

Nitrite and nitrate concentrations were determined by HPLC [8]. Monoterpene contents in the organic phase were determined with a Shimadzu GC-14A gas chromatograph equipped with a dual-column injector and two flame ionisation detectors and connected to a digital data-analysing system. Compounds were separated using an unpolar SE-54 column (0.32 $\mathrm{mm} \times 50 \mathrm{~m}, 0.5 \mu \mathrm{m}$ film thickness, Macherey-Nagel) and a polar $\mathrm{CW} 20 \mathrm{M}$ column $(0.32 \mathrm{~mm} \times 50 \mathrm{~m}, 0.5$ $\mu \mathrm{m}$ film thickness, Macherey-Nagel), hydrogen at a flow rate of $2 \mathrm{ml} \mathrm{min}{ }^{-1}$, and the following temper- ature program: injection port temperature, $250^{\circ} \mathrm{C}$; column temperature, $100^{\circ} \mathrm{C}$ for $2 \mathrm{~min}$, increasing to $220^{\circ} \mathrm{C}$ at a rate of $5^{\circ} \mathrm{C} \mathrm{min}^{-1}, 220^{\circ} \mathrm{C}$ for $4 \mathrm{~min}$; detection temperature, $280^{\circ} \mathrm{C}$. For gas-chromatography mass-spectroscopy, monoterpenes were separated on a NB-54 column $(0.32 \mathrm{~mm} \times 30 \mathrm{~m}, 0.25$ $\mu \mathrm{m}$ film thickness, Nordion) using helium as carrier gas and the following temperature program: injection port, $250^{\circ} \mathrm{C}$; column temperature $60^{\circ} \mathrm{C}$ for 2 min, increasing to $240^{\circ} \mathrm{C}$ at $10^{\circ} \mathrm{C} \mathrm{min}{ }^{-1}$; transfer temperature, $240^{\circ} \mathrm{C}$. Mass spectra were obtained with a MAT 8200 (Finnigan MAT) in the EI mode by using $70 \mathrm{eV}$, a scan speed of $1 \mathrm{~s}$ decade ${ }^{-1}$, and an ion source temperature of $200^{\circ} \mathrm{C}$.

\section{Results}

The degradation of linalool, geraniol and nerol was investigated in batch cultures of strain 47Lol to search for possible metabolic intermediates (Figs. 1-3). A linalool-limited denitrifying culture was used as inoculum to avoid the formation of transformation products from intracellular storage compounds. The cultures studied were nitrate-limited to include microbial transformations in the stationary phase whereby metabolic intermediates may be accumulated due to the lack of an electron acceptor.

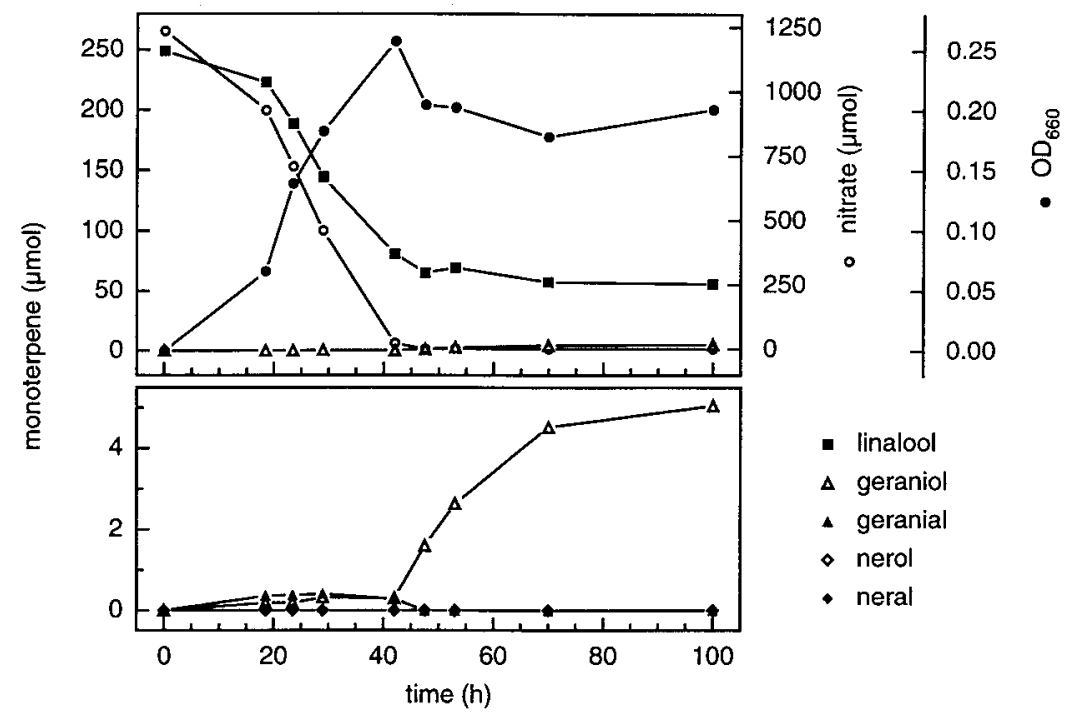

Fig. 1. Growth of strain 47Lol on linalool and nitrate (top). Small amounts of monoterpenes are shown in the enlarged graph (bottom). 


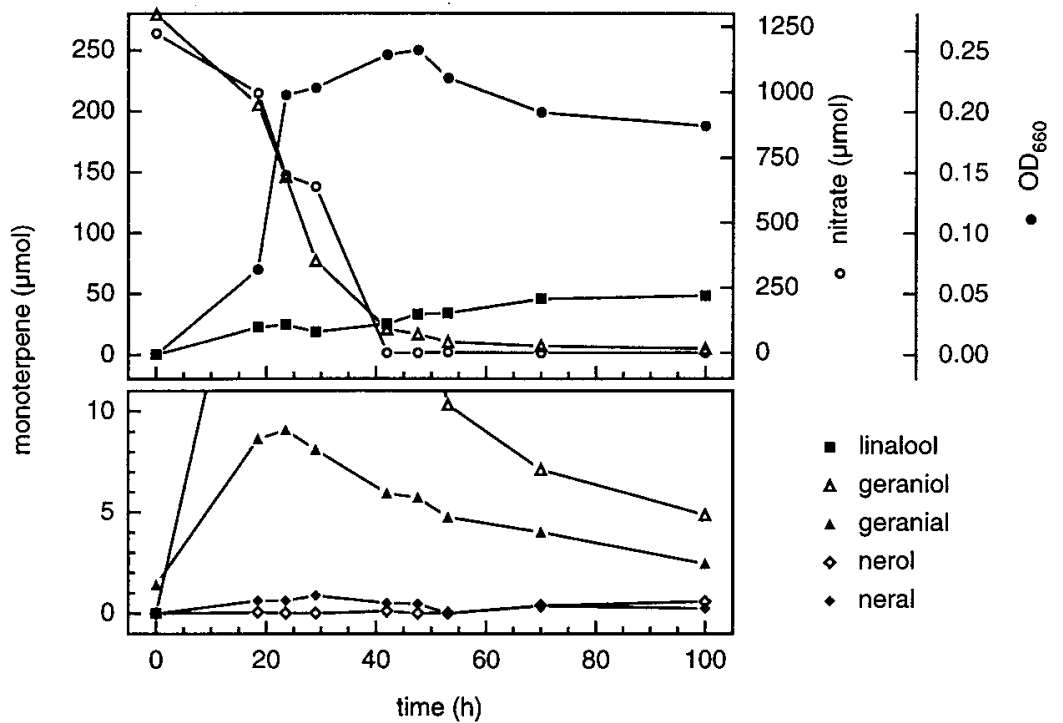

Fig. 2. Growth of strain 47Lol on geraniol and nitrate (top). Small amounts of monoterpenes are shown in the enlarged graph (bottom).

Linalool degradation (Fig. 1) proceeded initially without the formation of detectable amounts of geraniol (detection limit of monoterpenes: $0.2 \mu \mathrm{mol}$ culture $^{-1}$ ). Geraniol formation, but no nerol formation was observed upon nitrate depletion. Geraniol accumulated to a linalool:geraniol ratio of $11: 1$.

Geraniol utilisation (Fig. 2) was accompanied by formation of linalool, geranial and traces of neral already in the exponential growth phase of the culture. In the stationary phase, the amount of geraniol decreased further, geranial nearly disappeared and the linalool content increased further. The linalool: geraniol ratio increased throughout the experiment and reached a ratio of 10:1.

In contrast to linalool and geraniol, nerol was not completely mineralised by strain 47 Lol (Fig. 3). Ner-
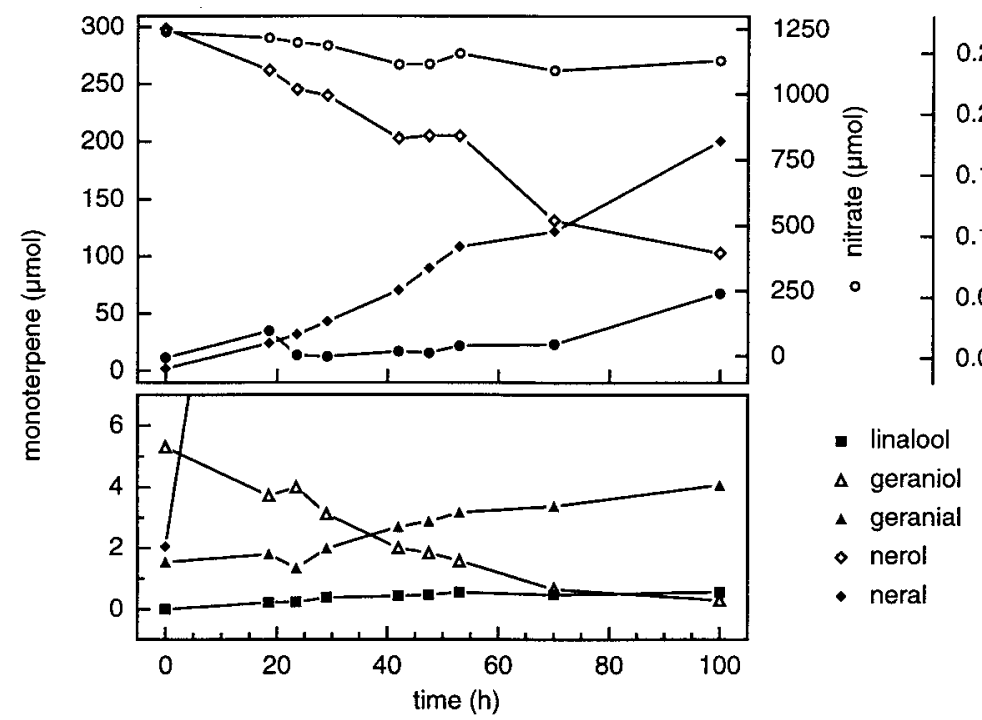

Fig. 3. Transformations of nerol by strain $47 \mathrm{Lol}$ (top). Small amounts of monoterpenes are shown in the enlarged graph (bottom). 


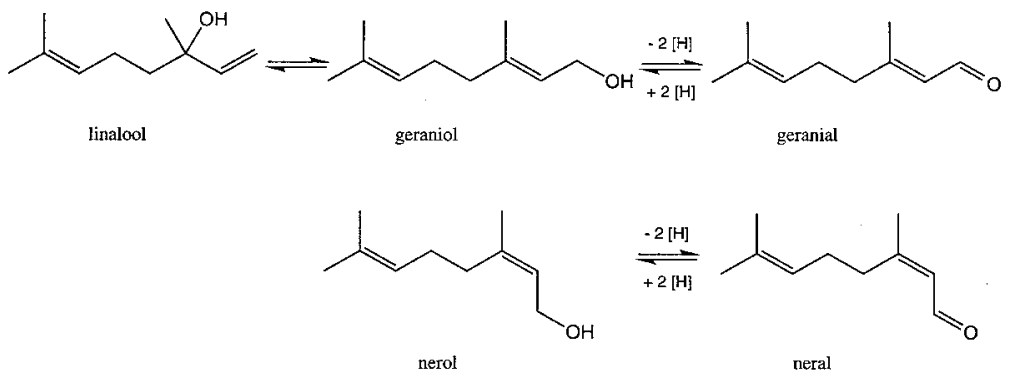

Fig. 4. Microbial transformations observed in cultures of strain 47Lol.

ol disappearance was paralleled by the formation of almost stoichiometric amounts of neral. Geraniol that was present as contaminant in nerol was evidently transformed to linalool and geranial. Only a small portion of nitrate was reduced. Biomass formation was not significant. The geraniol:geranial ratio in the stationary phase shifted from $2: 1$ in the nitrate-depleted geraniol culture (Fig. 2) to $1: 14$ in the electron donor-limited nerol culture (Fig. 3).

The identification of detected metabolites (linalool, geraniol, geranial and neral) was performed on two different GC-columns in comparison with authentic substances. Geranial and neral were commercially only available as mixture (citral). In addition, the aldehydes were not recovered from the polar carbowax column. Therefore, we confirmed our peak assignment by analysis of samples with gas-chromatography mass-spectroscopy (data not shown).

\section{Discussion}

In this study we found that strain 47Lol catalyses the isomerisation of linalool and geraniol regioselectively without formation of nerol. The presence of an oxidation product, geranial, suggests that the further biodegradation of geraniol occurs on the pathway described by Seubert [3,9]. In addition, nerol can be oxidised by strain 47Lol to neral Fig. 4. Neral may be considered a dead-end product that seems to inhibit also the geranial oxidation. Our results indicate that under the culture conditions used linalool is thermodynamically more stable than geraniol. The concentrations of linalool and geraniol measured in the heptamethylnonane phase indicate that linalool is $5.9 \mathrm{~kJ} \mathrm{~mol}^{-1}$ more stable than geraniol.
Enzyme-catalysed allylic rearrangements are well known in biochemistry, e.g. chorismate mutase, pyridoxal phosphate-dependent transaminations, keto/ enol tautomerisations and $\Delta^{5}$-3-ketosteroid isomerase [10]. Takigawa et al. reported the transitory formation of $\alpha$-curcumene during growth on $\alpha$-cedrene and proposed that $\alpha$-curcumene was formed by an allylic rearrangement of sec-cedrenol yielding an overall water elimination [11]. To our knowledge, a reversible enzymatic rearrangement of an allylic alcohol has not been reported. Further studies will aim at the characterisation of the enzymes involved in the linalool:geraniol isomerisation.

\section{Acknowledgments}

We thank Peter Schulze, Universität Bremen, for performing the gas chromatography-mass spectroscopy. This study was supported by the Deutsche Forschungsgemeinschaft and the Max-Planck-Gesellschaft.

\section{References}

[1] Zimmerman, P.R., Chatfield, R.B., Fishman, J., Crutzen, P.J. and Hanst, P.L. (1978) Estimates on the production of CO and $\mathrm{H}_{2}$ from the oxidation of hydrocarbon emissions from vegetation. Geophys. Res. Lett. 5, 679-682.

[2] Trudgill, P.W. (1984) Microbial degradation of the alicyclic ring. In: Microbial Degradation of Organic Compounds (Gibson, D.T., Ed.) pp. 131-180. Marcel Dekker, New York.

[3] Trudgill, P.W. (1994) Microbial metabolism and transformation of selected monoterpenes. In: Biochemistry of Microbial Degradation (Ratledge, C., Ed.) pp. 33-61. Kluwer, Dordrecht.

[4] Wood, S.E., Gaskin, J.F. and Langenheim, J.H. (1995) Loss 
of monoterpenes from Umbellularia californica leaf-litter. Biochem. System. Ecol. 23, 581-591.

[5] Bauer, K., Garbe, D. and Surburg, H. (1988) Flavors and fragrances. In: Ullmann's Encyclopedia of Industrial Chemistry, Vol. A 11: Fibers, 5. Synthetic Inorganic to Formaldehyde (Gerhartz, W., Ed.) pp. 141-250. VCH, Weinheim.

[6] Madyastha, K.M., Bhattacharyya, P.K. and Vaidyanathan, C.S. (1977) Metabolism of a monoterpene alcohol, linalool, by a soil pseudomonad. Can. J. Microbiol. 23, 230-239.

[7] Kieslich, K, Abraham, W.R., Stumpf, K., Thede, B. and Washausen, P. (1986) Transformation of terpenoids. In: Progress in Essential Oil Research (Brunke, E.-J., Ed.) pp. 367394. Walter de Gruyter, Berlin.
[8] Harder, J. and Probian, C. (1995) Microbial degradation of monoterpenes in the absence of molecular oxygen. Appl. Environ. Microbiol. 61, 3804-3808.

[9] Seubert, W. (1960) Degradation of isoprenoid compounds by microorganisms. I. Isolation and characterisation of an isoprenoid-degrading bacterium, Pseudomonas citronellolis $\mathrm{n}$. sp. J. Bacteriol. 79, 426-434.

[10] Schwab, J.M. and Henderson, B.S. (1990) Enzyme-catalysed allylic rearrangements. Chem. Rev. 90, 1203-1245.

[11] Takigawa, H., Kubota, H., Sonohara, H. Okuda, M., Tanaka, S., Fujikura, Y. and Ito, S. (1993) Novel allylic oxidation of $\alpha$-cedrene to sec-cedrenol by a Rhodococcus strain. Appl. Environ. Microbiol. 59, 1336-1341. 